\title{
A System to Measure Permeability of Unsaturated Soils using Local Pin-Type Sensors in Triaxial Apparatus
}

\author{
Jaylord U. Tan Tian ${ }^{\mathrm{i})}$, Junichi Koseki ${ }^{\mathrm{ii})}$, Hailong Wang ${ }^{\mathrm{iii}}$, Hiroyuki Kyokawa ${ }^{\text {iv) }}$, Takeshi Sato ${ }^{\text {v) }}$ \\ i) PhD Student, Department of Civil Engineering, University of Tokyo, 7-3-1 Hongo, Bunkyo-ku, Tokyo 113-8656 \\ ii) Professor, Department of Civil Engineering, University of Tokyo, 7-3-1 Hongo, Bunkyo-ku, Tokyo 113-8656 \\ iii) Asst. Prof., Waseda Research Institute for Science and Engineering, Waseda University, 3-4-1 Okubo, Shinjuku-ku, Tokyo $169-8555$ \\ iv) Asst. Prof., Department of Civil Engineering, University of Tokyo, 7-3-1 Hongo, Bunkyo-ku, Tokyo 113-8656 \\ v.) Senior Manager, Integrated Geotechnology Institute Ltd., 1-23-6, Yotsuya, Shinjuku-ku, Tokyo 160-0004
}

\begin{abstract}
This paper describes development of a triaxial permeameter apparatus for measuring coefficient of permeability of unsaturated soils by directly measuring the head difference between two points in a specimen using local pin-type sensors. The local pin-type sensors are made of stainless steel wrapped with membrane filter to measure suction. The apparatus includes the measurement of inflow rate by Mariotte's bottle and a weighing scale, while outflow rate is measured by burettes and a differential pressure transducer (DPT). Typical results show that the steady state flow condition can be achieved by using membrane filters to allow passage of water but prevent flow of free air. At steady state flow condition and reasonably stable measured head by the local pin-type sensors, permeability values were obtained by simple average.
\end{abstract}

Keywords: Permeability, Unsaturated Soils, Local Pin-Type Sensors, Triaxial Apparatus, Flexible Wall

\section{INTRODUCTION}

The coefficient of permeability, $k$ is essential for analyses of flow in unsaturated soils for applications in the geotechnical and geo-environmental areas. In general, there are two ways to determine $k$ of an unsaturated soil: direct and indirect techniques (Huang et al., 1998). For direct techniques, it can be further classified according to the flow mode as steady or unsteady state. In the steady state methods (also known as constant head method or constant flow method), a constant flow rate, $Q$ or hydraulic gradient, $i$ is applied under specific average water pressure head. Steady state is supposed to occur when flow rate of the soil specimen is equal upstream and downstream and if a constant $i$ is observed through the tested soil specimen (Masrouri et al., 2008). All methods assume the validity of Darcy's Law, as it also applies for the flow of water through unsaturated soils (Child et al., 1950; Fredlund et al., 1993). In steady state condition, Darcy's Law for laminar flow can be expressed as:

$$
k=\frac{Q}{A i}=\frac{Q}{A\left(\frac{\Delta h}{L}\right)}
$$

where $k(\mathrm{~L} / \mathrm{T})$ is the coefficient of permeability, $Q$ $\left(\mathrm{L}^{3} / \mathrm{T}\right)$ is the flow rate, $A\left(\mathrm{~L}^{2}\right)$ is the cross sectional area of the specimen, $\Delta h(\mathrm{~L})$ is the head difference or head loss between two points in the specimen, and $L(\mathrm{~L})$ is the length of the specimen between the points.

While $A$ and $L$ are geometrical properties of the specimen, $Q$ and $\Delta h$ are parameters induced by the fluid flow. Achieving steady state in testing on unsaturated soils is reported to be time consuming because equilibrium attainment may require several hours or days at each saturation level (Goh et al., 2015). Typically, a saturated high air entry ceramic disk is placed sandwiching the specimen, and this porous, ceramic disk allows the passage of water, but prevents the flow of free air (Fredlund et al., 1993). Continuity between the water in the soil and the water in the ceramic disk is necessary in order to correctly establish the matric suction.

Instead of ceramic disk, microporous membrane technology (referred as membrane filter in this paper) has been found out to have slightly higher hydraulic conductivity and can achieve much faster equilibrium time required than ceramic disk, hence, result in improved performance for the measurement or control of matric suctions at low range $(\sim 25 \mathrm{kPa})$ to avoid air diffusion (Nishimura et al., 2012; Wang et al., 2017).

In view of the above, a new system to measure permeability of unsaturated soils by measuring and expediting $Q$, and by directly measuring $\Delta h$ is presented in this paper. 


\section{TESTING MATERIAL}

Permeability tests used in this study were conducted on bauxite, which is a sedimentary rock with relatively high aluminum content, and is the principal ore of aluminum. Bauxite in this study has a specific gravity $\left(\mathrm{G}_{\mathrm{s}}\right)$ of 2.642 , median diameter $\left(\mathrm{D}_{50}\right)$ of $0.2 \mathrm{~mm}$, and fines of $40.6 \%$. Tests were conducted at degree of compaction of $80 \%\left(\mathrm{Dc}=\rho_{\mathrm{d}} / \rho_{\max }=80 \%\right)$. The particle size distribution is shown in Fig. 1.

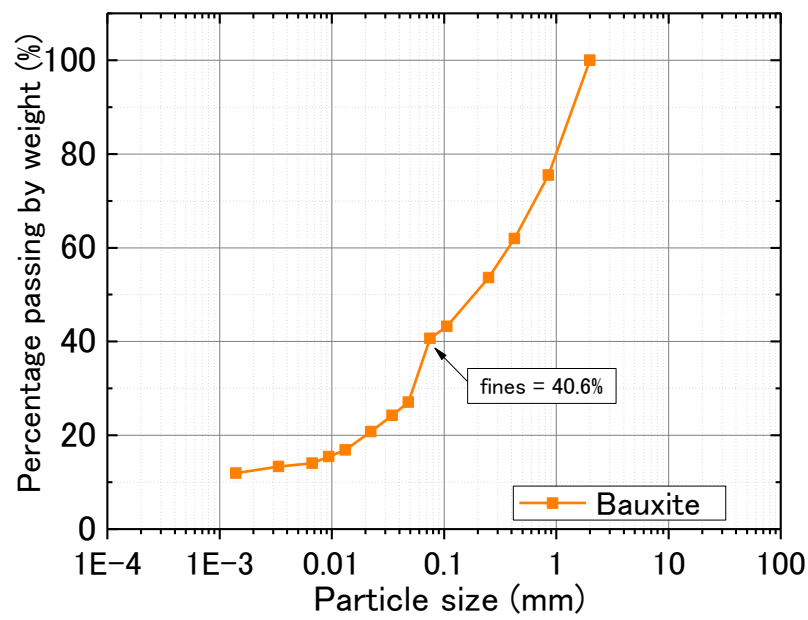

Fig. 1. Particle Size Distribution of Bauxite

The reason of interest in this material is because of maritime transport. In the process of loading the bulk cargoes into the ship and during transportation, the distribution of water contents on the bulk cargoes varies with respect to depth and changes with duration of travel. Liquefaction of bulk cargo can occur when excessive cyclic or dynamic loading, induced by rough seas and vessel vibrations, is transmitted into the cargo. One of the parameters in describing and understanding this phenomenon is the $k$ values of bauxite.

\section{TRIAXIAL PERMEAMETER APPARATUS}

In addition to rigid wall permeameters, flexible wall permeameters are also used in geotechnical engineering laboratories for determining $k$. There are several advantages of using flexible wall permeameters. The confining pressure can be applied, hence side wall leakage can be prevented, which commonly occurs in rigid wall permeameters. A study was conducted by Tan Tian et al. (2016) to investigate the side wall effects in a rigid mold. It was found out that in a rigid mold, the coefficient of permeability $k$, resulted to higher values due to side wall leakage effects. Another advantage of a flexible wall is that the stress-state variables of a soil specimen can be controlled during the experiment, making it possible to relate the permeability of soils to their stress states (Agus et al., 2003). In this experiment, a triaxial apparatus was utilized and a schematic figure is shown in Fig. 2, divided into three (3) sections: $I, I I, I I I$.

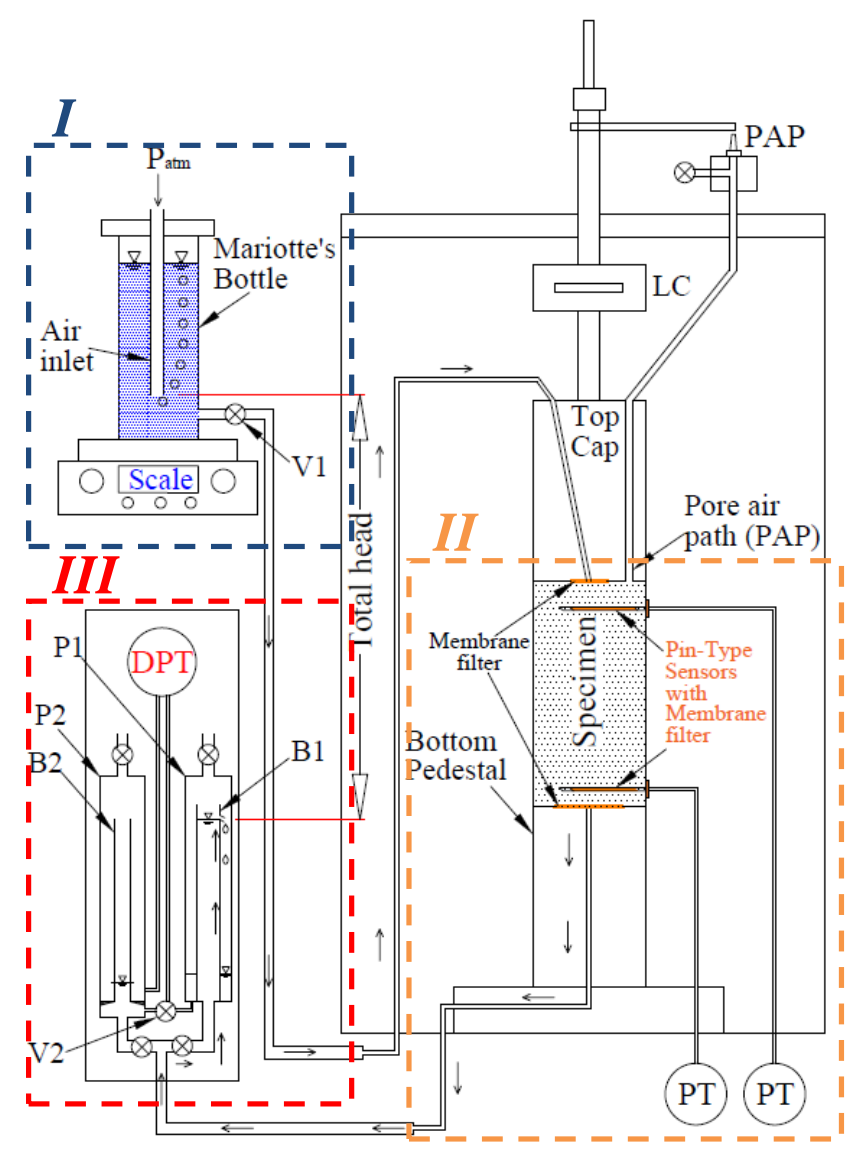

Fig. 2.Triaxial Permeameter System

\subsection{Section I Components}

This section has two main components namely, (1) Mariotte's bottle and the (2) weighing scale.

Mariotte's bottle is a device that delivers a constant pressure from closed bottles or tanks. As shown in the schematic diagram in section $I$, a reservoir is supplied with an air inlet. The pressure at the bottom of the air inlet is always the same as the pressure outside the reservoir, i.e. the atmospheric pressure $\left(\mathrm{P}_{\mathrm{atm}}\right)$. The Mariotte's bottle used in the experiment was designed to supply a total volume of approximately $80 \mathrm{ml}$ to the specimen.

The weighing scale is a commercial scale which has sensitivity up to the hundredth decimal. It is accompanied with USB port which can be connected to the computer (RS-232C interface) and the data can be collected.

\subsection{Section II Components}

This section is composed of the (1) top cap and bottom pedestal, (2) membrane filter, and (3) local pin-type sensors (connected to pressure transducers (PT)).

The specimen is mounted at the bottom pedestal and connected to the top cap, which is connected to load cell (LC). Fig. 3 shows the detail of the bottom pedestal and the top cap. The top cap has a pore air path (PAP) so that the specimen is connected to the atmospheric 
pressure in the duration of the test. Instead of ceramic disk, membrane filter was implemented in this set-up. Membrane filters were secured on both bottom pedestal and top cap by stainless covers.

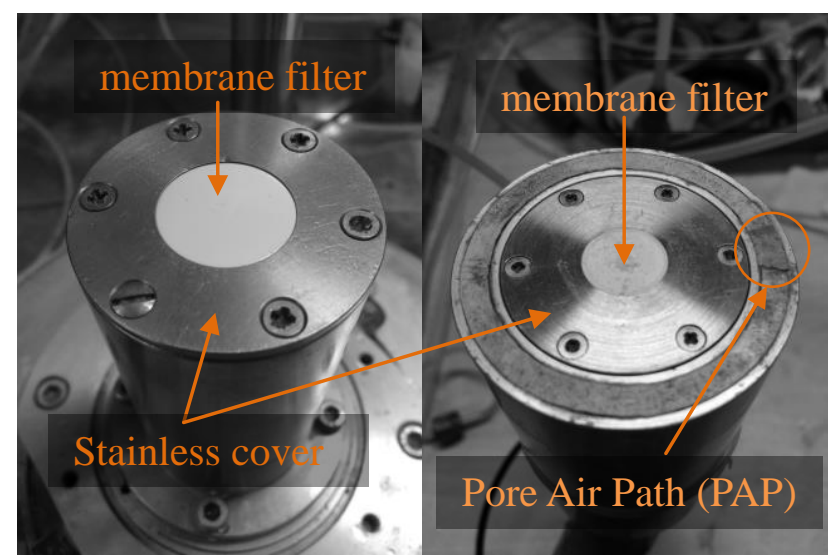

Fig. 3.bottom pedestal (left fig.) and top cap inverted (right fig.)

The membrane filters used in this study were manufactured by Pall Corporation. Membrane type 450 has pore size of $0.45 \mu \mathrm{m}$, thickness of $140 \mu \mathrm{m}$, air entry value $(\mathrm{AEV})$ of $250 \mathrm{kPa}$. Further details of the membrane filter can be read in Nishimura et al., (2012) and Wang et al., (2017).

In order to measure $\Delta h$ directly, local pin-type sensors were used. The local pin-type sensor is made of a hollow stainless steel with $0.15 \mathrm{~cm}$ diameter initially. It is then modified by cutting it on the side and crafting its edges to have a pointed side for piercing. The end product is a $3.5 \mathrm{~cm}$ long pin wrapped with membrane filter (same membrane type as the one used in bottom pedestal and top cap). The sensors are connected independently to their respective pressure transducers (PT). Fig.4 shows the local pin-type sensor.

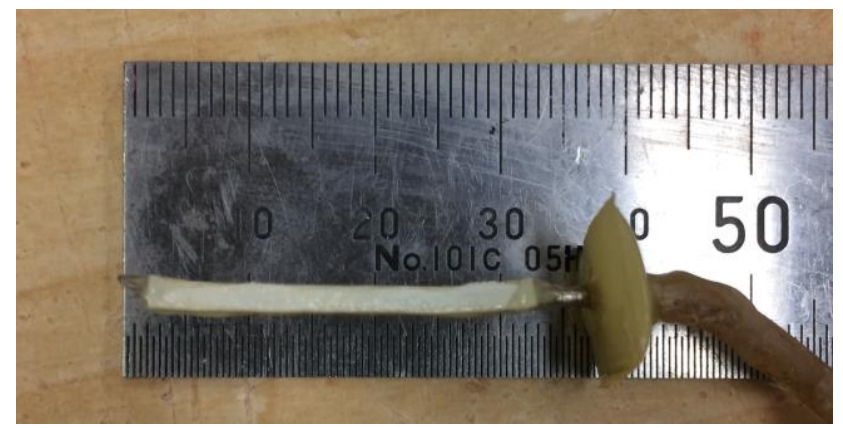

Fig. 4.Local Pin-Type Sensor $3.5 \mathrm{~cm}$ long

\subsection{Section III Components}

This section is composed of two pipes, P1 and P2. P1 contains burette B1, which is the downstream of the constant head supplied to the specimen. The overflow of B1 is the outflow of the fluid from the specimen and measurement of flow rate is determined by a differential pressure transducer (DPT).

\section{EXPERIMENTAL PROCEDURE}

\subsection{Pre-Seepage Process}

All specimens have dimension of $5 \mathrm{~cm}$ in diameter and $10 \mathrm{~cm}$ in height. A pre-determined amount of water was mixed with oven-dried bauxite in order to achieve the equivalent target/desired test degree of saturation $\left(\mathrm{S}_{\mathrm{r}}\right)$. After which, it was statically compressed to the desired dry density in a rigid mold.

Prior to the seepage, the bottom pedestal and top cap were pre-saturated before the specimen was even placed on it, while membrane filter was also saturated separately before placing and securing it on the pedestals.

The rubber membrane was pierced at two points, 8 $\mathrm{cm}$ apart along the vertical direction (Fig. 5, left figure). It was to indicate where the local pin-type sensors must be inserted once the specimen is mounted on the pedestal. The specimen was placed carefully on the rubber membrane before placing it on the pedestal. Once the specimen was secured at the pedestals, a back pressure of $5 \mathrm{kPa}$ was applied to sustain the specimen, before local pin-type sensors were inserted. Fig. 5 (right) shows the specimen on pedestals with inserted local pin-type sensors.

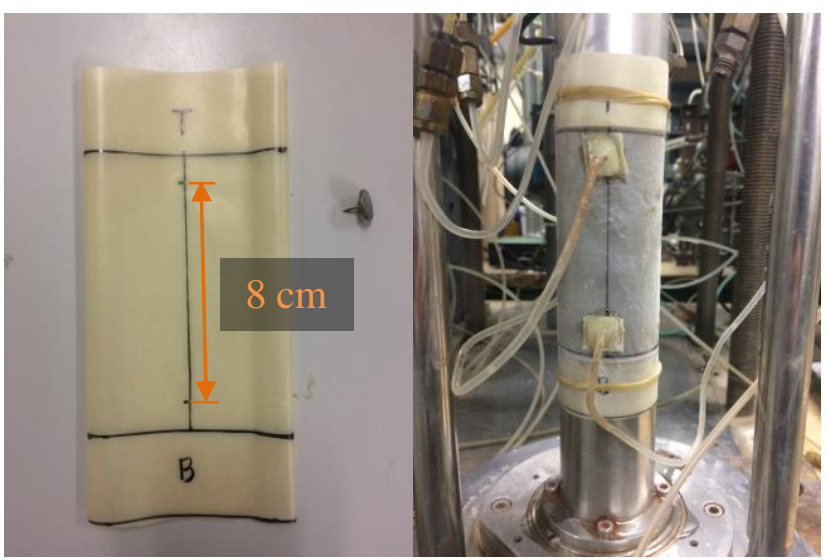

Fig. 5. rubber membrane (left) and inserted local pin-type sensors on the specimen (right)

Prior to the seepage of water to the specimen, it is essential to apply the desired confining stress to the specimen. Specimens were consolidated for 2 hours.

\subsection{Seepage Process}

Before conducting the seepage step, it was first ensured that the negative pressure applied at the burette in section III corresponded to the suction measured at the membrane filter from the bottom pedestal and top cap. Otherwise, the fluid cannot flow from the specimen to pipe $\mathrm{P} 1$.

Referring to the schematic diagram in Fig.2 the seepage flow can be explained in three sections.

In section $I$, valve V1 allows the water to enter through the specimen. Once V1 is turned open, Mariotte's bottle will always supply the water at 
atmospheric pressure at the bottom of the air inlet and will deliver a flow under constant head height, regardless of the changing water level within the reservoir. The change in mass of the Mariotte's bottle due to volume change was obtained by the weighing scale. One can specify the interval of data collection with a minimum of $1 \mathrm{sec}$. Inflow rate can then be obtained by the change in volume (change in mass) per time interval.

The fluid flow direction was from top to bottom of the specimen as it flows through section II. Pore air path (PAP) was exposed to $\mathrm{P}_{\mathrm{atm}}$, hence pore air pressure $\left(u_{a}\right)$ was kept zero throughout the experiment. The fluid flowing through the specimen caused some change to the pressures measured by the pressure transducers (PT) on the local pin-type sensors. The pressure difference was converted to head difference to acquire the head difference of the local pin-type sensors with respect to time.

In section III, the overflow from B1 would naturally accumulate water in the outer burette of $\mathrm{P} 1$. The change in volume was measured by the DPT between the accumulation of water with respect to the reference level at B2. From the volume change, the outflow rate can be obtained with respect to its time interval.

Seepage was stopped once the inflow rate was assessed to be the same as outflow rate and the measured head difference of the local pin-type sensors was reasonably stable.

\subsection{Post-Seepage Process}

After the seepage was stopped, the recording of head difference of the local pin-type sensors was continued. The real head difference was assessed to be the decrease of the head difference when fluid was no longer flowing through the specimen.

\section{TYPICAL TEST RESULTS}

Typical test result is represented by a specimen prepared at initial saturation degree of $65 \%\left(\mathrm{Sr}_{\text {ini }}=65 \%\right)$. In Fig.6, the water inflow and outflow were continuously monitored until a steady-state condition was established when the inflow rate was approximately equal to the outflow rate.

It can be observed that the flow of water seeped through the specimen at a relatively short elapsed time from inflow to outflow. At approximately $1500 \mathrm{sec}$, the difference of the inflow rate and outflow rate started to exhibit equivalent values, although it cannot be said to have achieved steady state yet since the flows were still decreasing. The steady state of the typical plot shown was achieved at about $10500 \mathrm{sec}$ (marked by a vertical solid line), when the flow difference was approximately zero and the inflow rate and outflow rate were both approaching horizontal (marked by a horizontal solid line.

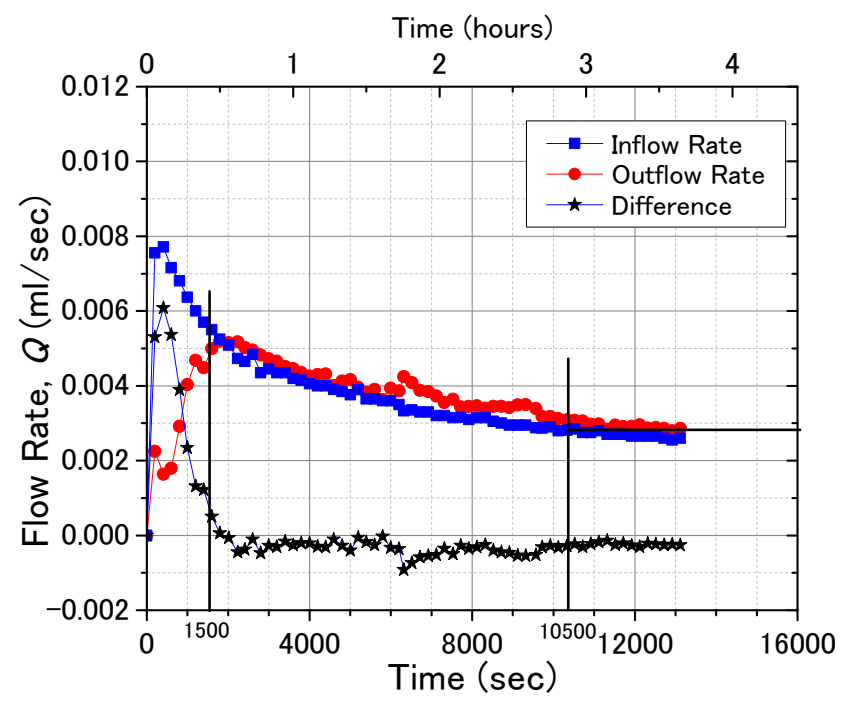

Fig. 6. Typical plot of the steady state flow condition

The head difference $(\Delta h)$ is measured by the difference of the pressures of the top and bottom local pin-type sensors converted to pressure heads. It can be said from Fig.7 that the head difference measured was reasonably stable from about $1500 \mathrm{sec}$ mark. This is consistent with the information that can be drawn from Fig.6 since initially, when inflow of water surged to a comparatively higher flow rate, the top local pin-type sensor became more saturated first (due to flow direction from top to bottom). Hence, the pore water pressure $\left(u_{w}\right)$ increased, resulting to lower suction values. This implied lower equivalent head value for the top local pin-type sensor, but resulted to an increased $\Delta h$. As the water seeping through the specimen affected both the top and bottom local pin-type sensors with respect to time, it achieved a relatively stable $\Delta h$ at a certain time when the water seeping through the specimen had found its path. Although the head was reasonably stable from approximately $1500 \mathrm{sec}$, the data obtained from 10500 sec and beyond were treated as the $\Delta h$ at steady-state condition.

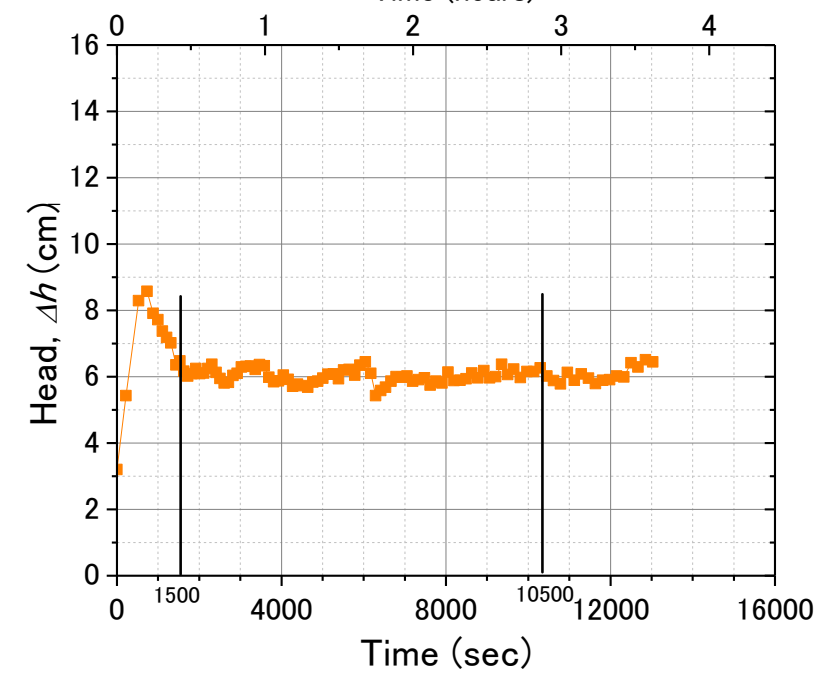

Fig. 7. Typical plot of the head difference measured by the local pin-type sensors 
From the flow rate time history and head difference time history, the coefficient of permeability can be obtained from Equation 1. Fig. 8 shows the corresponding $k$ vs time history from the typical test. Consequently from Fig.6, the $k$ values at steady state condition was also obtained from the $10500 \mathrm{sec}$ mark. The $k$ value for this test was taken to be the simple average of those values (indicated by an ellipse in Fig.8), which is equal to $k_{\text {avg }}=2.47 \times 10^{-4} \mathrm{~cm} / \mathrm{sec}$.

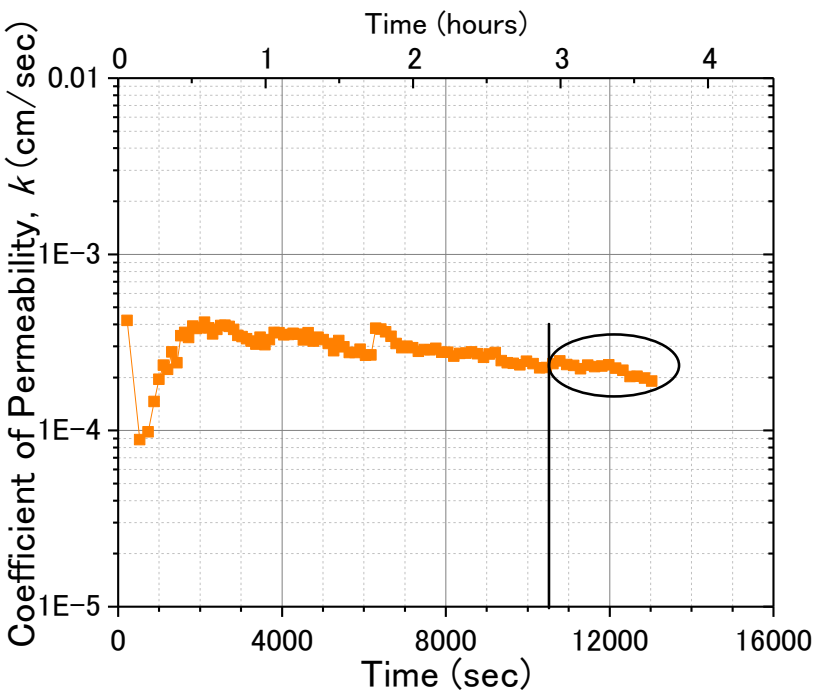

Fig. 8. Corresponding $k$ vs time plot

Fig. 6-8 are plots that could be obtained under seepage condition in the specimen. The head difference was continually measured even after the seepage was stopped. Fig. 9 shows the typical behavior of the head difference at the post-seepage process.

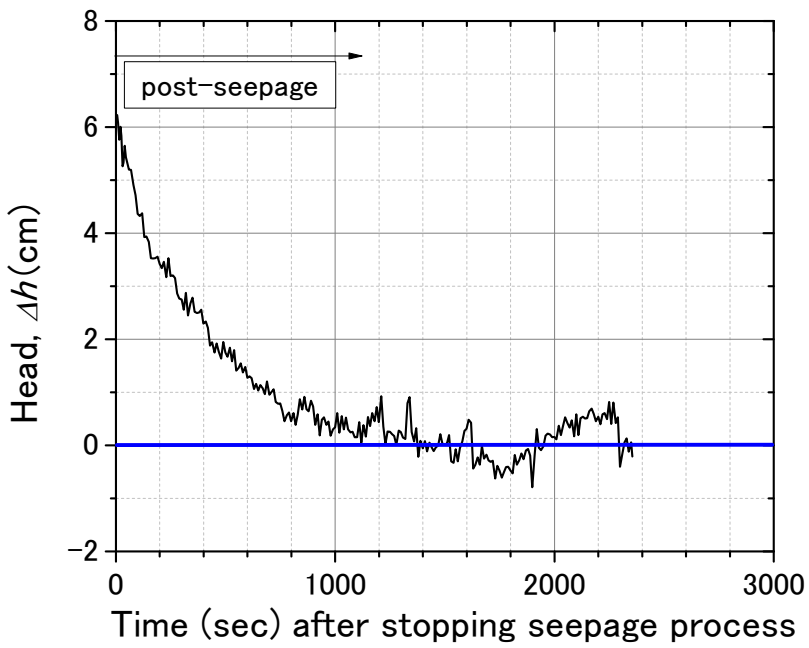

Fig. 9. Typical plot of the head difference measured by the local pin-type sensors at post-seepage

The head difference was expected to be zero once seepage had been stopped. Depending on the duration of the test, the inevitable fluctuation of the transducers and the imperfections of fully-saturating the local pin-type sensors caused the pins to respond differently after a long period of time. For this particular example, there was no need to correct Fig.7 since the post seepage data suggested that the head difference was zero once the seepage was stopped.

\section{CONCLUSIONS}

A new triaxial permeameter system was presented in this paper, with the following features of the system:

(1) Inflow rate was measured by Mariotte's bottle and a weighing scale, while outflow rate was measured by burettes and differential pressure transducer. Steady state flow condition can be achieved by using membrane filters to facilitate flow of water but prevent flow of free air.

(2) Unsaturated permeability could be evaluated using the newly developed local pin-type sensors to measure the head difference directly. Permeability values were obtained by simple average at steady state flow condition and measured head is reasonably stable.

\section{REFERENCES}

1) Agus,S.S., Leong,E.C., Rahardjo,H. (2003). A flexible wall permeability for measurements of water and air coefficient of permeability of residual soil. Canadian Geotechnical Journal. 40, 559-574.

2) Childs EC, Collis-George N (1950). The permeability of porous materials. Proceedings of the Royal Society 201A:392-405

3) Fredlund DG, Rahardjo H (1993). Soil mechanics for unsaturated soils. Wiley, New York

4) Goh, S.G. \& Rahardjo, H \& Leong, E. (2015). Modification of triaxial apparatus for permeability measurement of unsaturated soils. Soils and Foundations. 55(1), 63-73

5) Huang,S., Fredlund, D.G., Barbour, S.L. (1998). Measurement of the coefficient of permeability for a deformable unsaturated soil using a triaxial permeameter. Canadian Geotechnical Journal. 35, 426-432.

6) Masrouri, F., Bicalho K.V., Kawai, Katsuyuki (2008). Laboratory Testing in Unsaturated Soils. Geotechnical and Geological Engineering. 26, 691-704.

7) Nishimura, T., Koseki, J., Fredlund, D.G., and Rahardjo, H. (2012) Microporous membrane technology for measurement of soil-water characteristic curve. Geotechnical Testing Journal, 35: 201-208. doi:10.1520/GTJ103670.

8) Tan Tian, J., Koseki, J., Wang, H., Sato, T. (2016). Local Measurement of the Permeability of Sands using Pin-Type Sensors in the Triaxial Apparatus. The 18th International Symposium, CS2-029. JSCE Conference. Sendai, Sept 08 2016

9) Wang, H., Koseki, J., Cai, F., Nishimura, T. (2018). Membrane filter properties and application of the filter to undrained cyclic triaxial test of unsaturated materials. Canadian Geotechnical Journal, 55 (9): 1349-1357. https://doi.org/10.1139/cgj-2017-0480 